\title{
La interseccionalidad en las políticas migratorias de la Comunidad de Madrid
}

\author{
Interseccionality in the migration policies of the Community of \\ Madrid
}

Cory Marcela Duarte Hidalgo

\begin{abstract}
Resumen
El artículo pretende revisar el concepto de interseccionalidad a partir del análisis de marcos de política realizado a los planes de integración de personas migrantes de la Comunidad de Madrid. El artículo da a conocer la existencia de marcos interpretativos de política en los que las mujeres migrantes son representadas como vulnerables, subalternas y homogeneizadas. Así, se promueve una ciudadanía excluyente, que margina a las mujeres migrantes subrayando la subalternidad que se les atribuye.
\end{abstract}

Palabras clave: migración - feminismos - mujeres migrantes - interseccionalidades - marcos interpretativos de política.

\begin{abstract}
This article aims review the concept of intersectionality, from de analysis of policy frameworks made to plans for migrants' integration in the Community of Madrid. The article discloses the reality of policy framing where those migrant women are represented as vulnerable, subordinate and homogenized. Therefore, these policies promote an exclusionary citizenship, which excludes migrant women, emphasizing the subordination assigned to them.
\end{abstract}

Key words: migration - feminism - migrant women - intersectionalities - policy framing.

Fecha de recepción: 26 de abril de 2012

Fecha de aprobación: 20 de mayo de 2013

1 Doctoranda en Trabajo Social, Universidad Complutense de Madrid. Máster en Estudios Feministas, Máster en Trabajo Social comunitario por la Universidad Complutense de Madrid y Máster en Inmigración, refugio y relaciones intercomunitarias, Universidad Autónoma de Madrid. Asistente Social (UTEM). Directora Departamento de Trabajo Social, Universidad de Atacama.coryduarte@gmail.com 


\section{LA INTERSECCIONALIDAD EN LAS POLÍTICAS MIGRATORIAS: EL CASO DE LA COMUNIDAD DE MADRID ${ }^{2}$}

\section{Presentación}

El artículo que en este apartado se introduce da a conocer una investigación realizada durante el año 2011, la que tuvo por objetivo principal el identificar los principales marcos de política presentes en los planes de integración de población migrante efectuados por la Comunidad de Madrid (2006-2008 y 2009-2012) a la luz del concepto de interseccionalidad.

La comunidad antes mencionada es la autonomía con la mayor recepción de población migrante en España. Antes de la crisis actual, las cifras indicaban que una quinta parte de las personas empadronadas estaban registradas en la Comunidad de Madrid (CAM, 2006; 2009).

Ante la envergadura del fenómeno migratorio, se diseñaron planes de integración, instaurando en ellos distintas estrategias para la población migrante establecida en dicha comunidad. Esto resulta fundamental para el cometido que tiene la Administración local, ya que esta institucionalidad ejerce un papel esencial en el objetivo final de facilitar la integración social de las personas migrantes mediante el desarrollo de una política de recepción, acogida y de acompañamiento que intente garantizar la cohesión social y la convivencia intercultural dentro del territorio (Camós, 2006: 21).

El análisis de los planes a partir del análisis de marcos de política, permite dar a conocer la existencia de marcos interpretativos presentes en las acciones implementadas por la Comunidad de Madrid respecto de la población migrante. Sin embargo, y para efectos del estudio, solo se analizaron las interpretaciones, construcciones e imágenes con las que son representadas las mujeres de origen extranjero. Esto dará cuenta de la presencia de marcos interpretativos, pero también de la idea de ciudadanía presente en las planificaciones de dicha comunidad autónoma.

Sin adelantar mucho más, exponemos la secuencia de este artículo: en un primer apartado se revisan algunos puntos respecto de la migración de las mujeres. Posteriormente, el documento se centra en las críticas que el movimiento feminista ha recibido debido a la homogeneización del concepto "mujer". Estas críticas dan pie a la construcción de la idea de intersecciones entre las diferentes desigualdades. La noción de interseccionalidad es revisada por autoras como Knudsen, Anthías, Verloo,

\footnotetext{
El artículo se basa en la investigación realizada como trabajo final del Máster en Estudios Feministas de la Universidad Complutense de Madrid. Por tanto, corresponde aquí mencionar y agradecer el crucial acompañamiento y dirección de la profesora Emanuela Lombardo, quien tutorizó el trabajo de investigación. La profesora Lombardo fue parte del equipo de investigadoras del Departamento de Ciencia Política y de la Administración de la UCM que participó en el proyecto europeo MAGEEQ, Mainstreaming Gender Equality in Europe, V Programa Marco de la Unión Europea. La metodología utilizada en dicho proyecto fue adaptada para la realización de esta investigación.
} 
Lombardo y Lugones. Finaliza el apartado referencial la exposición de la idea de marcos de política.

Posteriormente se expone el método utilizado para el análisis de textos políticos, a base de lo realizado por el proyecto europeo Mainstreaming Gender Equality in Europe, V Programa Marco de la Unión Europea (MAGEEQ).

El artículo finaliza con los resultados del análisis y la descripción de las principales conclusiones de este estudio. Luego de esto se exponen algunas reflexiones sobre la necesidad de incluir la noción de interseccionalidad en los estudios feministas y en las políticas sociales.

\section{Algunos apuntes a considerar respecto de la migración de mujeres}

Para comenzar, debemos señalar que desde tiempos remotos las mujeres han participado en los procesos migratorios, sin embargo, su movilización ha sido desvalorizada, invisibilizada y por tanto no cuantificada. Lo anterior no tiene relación con el poco estudio de la cuestión, sino más bien con el escaso impacto que este tema tiene en los medios de comunicación de masas y en las agendas legislativas (Plataforma Interamericana de Derechos Humanos, Democracia y Desarrollo, 2008: 31).

Una de las explicaciones de la aparente invisibilización de las mujeres migrantes es el que no se le ha entregado suficiente valor a los estudios sobre migraciones femeninas. Las razones estarían vinculadas a que este tipo de trabajos han sido realizados, mayoritariamente, por mujeres investigadoras; pero también, por el hecho de que las investigaciones sobre migraciones "han sido sesgadas a la visión masculina de la movilidad" (2008: 32).

En respuesta a lo anterior, se ha producido un cambio en el discurso migratorio que en los últimos años sí ha visibilizado la participación de las mujeres en las migraciones. La preocupación desde los centros universitarios y científicos de sacar de la invisibilidad a las mujeres migrantes ha generado una reivindicación de su rol como "actrices económicas y sociales", lo que ha generado una percepción equívoca respecto de la feminización de las migraciones, fenómeno que puede percibirse "más acentuado de lo que realmente muestran los datos" (Oso, 2007: 650).

La migración de mujeres está asociada al desempeño en las sociedades de acogida de tareas relacionadas con labores reproductivas y de cuidados. Esta situación refuerza la división sexual del trabajo y los roles tradicionales, desencadenando correcciones en las desigualdades de género en las sociedades receptoras a costa de reproducirlas a escala internacional (Alonso, 2011). La principal característica de la migración laboral femenina es que se basa en "Ia reproducción y explotación de las desigualdades de género por parte del capitalismo global" (Orozco, Paiewonsky y García, 2008). 
Diversos estudios han puesto de manifiesto que con la incorporación de las mujeres de los países del Norte al mercado laboral, el trabajo no remunerado que ellas realizaban, el llamado reproductivo, es transferido a otras mujeres, generalmente migrantes, creándose un sector "poco reconocido, con reglas no siempre claras y por lo tanto escasamente regulado, imponiendo límites en el acceso a derechos u otros sectores económicos con mejores condiciones laborales" (Robert y López: 2008). La demanda de servicios asociados a los cuidados en los países ricos requiere de personas que cubran puestos de trabajo ubicados en posiciones tradicionalmente menos valoradas, entre las que encontramos el servicio doméstico, el servicio de cuidado personal y los servicios sexuales (Anthias y Lazaridis: 2000, citado por Oso, 2007: 652).

Asimismo, se ha puesto atención sobre la vinculación de las migraciones con la necesidad de cubrir este tipo de puestos de trabajo precarios, feminizados, mal remunerados y desvalorizados socialmente (CEPAL, 2006; Carrizo, 2006; Parella, 2005). Los estudios han señalado que las condiciones laborales de las mujeres migrantes están entrelazadas con elementos como el sexismo, racismo y la servidumbre (Colectivo IOE, 2001; Parella, 2005; Solé, 2009). A esto se añaden las lógicas del Estado-Nación y del diferencialismo cultural, de forma que "su posición depende, objetivamente, del lugar en que se encuentren respecto del modelo dominante: varón, de elevada posición socioeconómica, con plenos derechos de ciudadanía, blanco y adscrito a la cultura dominante". (Colectivo IOÉ, 1998). Es por esto que la migración femenina no puede ser abordada si no es bajo la consideración de las dimensiones de género, etnia y raza (Parella, 2003).

Cabe destacar que no se puede reducir la migración a un punto de vista meramente económico o laboral, desvinculando del proceso migratorio cualquier otra dimensión (Pedone, 2004). Temas como las cadenas de cuidado, las comunidades trasnacionales, las remesas, el tráfico sexual y la violencia, son solo algunas de las dimensiones a considerar en la revisión de la migración de las mujeres.

\section{La mirada homogeneizada de la mujer v/s las diversidades de mujeres}

Las investigaciones feministas, así como el propio movimiento, no han sido ajenas a una serie de críticas y cuestionamientos en torno a la concepción homogenizada que se tenía sobre la categoría "mujer". Las críticas estaban orientadas a que el movimiento feminista respondía a una imagen de mujer occidental, blanca, burguesa y heterosexual, que no hacía eco de la diversidad de mujeres y de las situaciones distintas de opresión y dominación que estas padecían en sus contextos particulares (Lorde, 2003; Hooks, 2004; Lugones, 2008; Spivak, 2010). Se postulaba así que el feminismo había nacido "con una pretensión de universalismo semejante al que le ha excluido" (Suárez Navas, 2008: 44).

Con este tipo de cuestionamientos, se apuntaba a la existencia de una mirada homogeneizante de "las mujeres" discordante con las realidades de mujeres racializadas y etnizadas. La definición de la categoría "mujer" se había realizado desde posiciones de privilegio de raza, clase y sexualidad (Curiel, 2011). De esta manera, se intentó unificarlas a base de una configuración identitaria construida solo en torno a las diferencias 
de género (Trujillo, 2011: 64) y en la que existía una "reducción del conglomerado de estructuras de dominación haciendo la sexual la determinante" (Suárez Navas, 2008: 46). Esta unificación de la identidad se basa en homogeneizar las mujeres (Lorde, 2003: 124) omitiendo su ubicación "dentro de múltiples y frecuentemente poco armónicas agendas" (Haraway, 1995: 190).

Los feminismos de color criticaron los binarismos y la lógica de las fronteras presentes en el "feminismo tradicional". Planteaban que a partir de un deseo de control y de pureza se había generado un rechazo a todo aquello que es múltiple y que no puede ser clasificado (Lugones, 1994). Los feminismos a los cuales se hace referencia recogieron las identidades mestizas, móviles y siempre en problemas (Anzaldúa, 1987) rescatando la existencia de tierras medias. Los saberes provenientes de estos espacios generan la idea de una gnosis fronteriza, configurándose un conocimiento desde una perspectiva subalterna, concebido desde los límites, desde las fronteras del sistemamundo, moderno/colonial (Mignolo, 2003).

Desde estas visiones se denuncia que todas las categorías comprensivas y constitutivas de la historia y de la identidad de las mujeres se han construido solo desde un punto de vista de Occidente (Spivak, 2010). Se acusa al "feminismo tradicional" de entablar discusiones sobre teoría feminista "sin entrar a analizar nuestras numerosas diferencias y sin considerar espacios a las significativas aportaciones de las mujeres pobres, negras, del tercer mundo y lesbianas" (Lorde, 2003: 115). Esta perspectiva a todas luces hegemónica pretendía "reducir el sujeto-objeto de conocimiento a una concepción estática y homogénea", en contraste con una mirada diversa y no excluyente, ya que solo a partir de un ejercicio que permita el "reconocimiento de las diferencias existente entre los distintos perfiles y colectivos de mujeres podremos abordar adecuadamente el modo en que se construyen y articulan esas experiencias" (Reigada, 2008: 103).

Los feminismos periféricos hacen hincapié en que las mujeres han estado sujetas a procesos culturales y políticos desencadenados por el sistema capitalista y la modernidad occidental. Asimismo, estos feminismos entregan gran relevancia a la necesidad de reconocer tanto las producciones teóricas como las prácticas subordinadas que se han hecho desde los márgenes (Curiel 2011: 200). Los feminismos periféricos postcoloniales o del tercer mundo evidencian la(s) realidad(es) de la diversidad de mujeres, de sus agencias, y sus capacidades de actuación. Se reclama el que se "consideren y nombren las diferentes diferencias entre las propias mujeres, es decir, las existentes dentro de la identidad colectiva articulada por el feminismo" (Trujillo, 2011: 165). Así también, se plantea la necesidad de analizar "las causas que producen las diferencias de clase, raza, etnia, opción sexual o migración, y tener en cuenta cómo las experiencias de esas diferencias afectan la forma de ser mujeres" (2011: 166).

Se levantan críticas respecto de que las opresiones no se dan en un estado puro, sino más bien se combinan y potencian, por tanto se hace necesario la articulación de las múltiples opresiones como variables dependientes, "porque cada una inscribe en las otrasy es constitutiva de y por las otras" (Brah, 2004). La consideración de las distintas categorías en 
los análisis feministas permitió estudios que dieran cuenta de las intersecciones entre las desigualdades en que se mueven y superviven las mujeres (Hancok, 2007; Anthías, 2011; Crenshaw, 2003; Verloo, 2005). En este sentido, los estudios sobre las interseccionalidades permitieron considerar las identidades múltiples de los y las sujetos, visibilizando que no se es solamente mujer, si no que se es en un contexto y circunstancias específicas.

\section{La noción de interseccionalidad}

Para adentrarnos en esta idea, es preciso reconocer que los feminismos periféricos lograron poner énfasis en que "la discriminación de género no es adicional sino relacional, lo que significa que colorea y es coloreada por otras divisiones sociales, como la clase social y la raza (o etnicidad)" (Rodríguez, 2011: 20).

Las desigualdades de género se solapan con otras jerarquías sociales, y las posiciones sociales de las mujeres han de ser comprendidas en la intersección de las distintas categorías (Peterson, 2007), estas diferencias debiesen ser reconocidas sin llegar a esencializar a las mujeres con la finalidad de construir alianzas contra todas las formas de discriminación (2007: 41).

En este sentido, Mohanty plantea que la experiencia de ser mujer "puede crear una unidad ilusoria, porque no es la experiencia de ser mujer, sino el significado atribuido al género, la raza, la clase y la edad en diversos momentos históricos los que tiene significación estratégica" (2002: 101). Y esa misma significación estratégica permite plantear los temas que se entrelazan en el ejercicio teórico y práctico de las diversas categorías, ya que si no "¿Cómo se negocia entre mi historia y la tuya? ¿Cómo se entretejen nuestras diferencias y cómo de hecho se organizan jerárquicamente?" (2002: 91).

Knudsen, en 2006, sitúa en la década de los noventa el surgimiento del concepto de interseccionalidad. Sin embargo, los cruces entre categorías ya eran apreciados en la segunda ola del movimiento feminista, pero también, en los feminismos poscoloniales y en la teoría queer. En este sentido, las investigaciones feministas han cambiado el enfoque de forma paulatina: "partiendo de acentuar la igualdad entre hombres y mujeres, para más tarde enfatizar las diferencias de género y llegando a destacar la diversidad de género y la «interseccionalidad»" (Squires, 1999, citada en Peterson, 2007: 41).

La importancia de las relaciones de interdependencia entre las distintas desigualdades han estado presentes en los análisis teóricos de algunas feministas, entre las que destaca Crenshaw. Esta autora afirma que las estrategias dirigidas a una desigualdad no son neutrales a las demás (Crenshaw, 1999), lo que provoca la reflexión acerca de las "dinámicas de privilegios y exclusiones que emergen cuando no se considera la atención a las personas que se encuentran en el punto de intersección de las distintas desigualdades" (Lombardo y Verloo, 2010: 14).

María Lugones plantea que la"interseccionalidad revela lo que no se ve cuando categorías como género y raza se conceptualizan como separadas unas de otras" (79: 2008). Los 
intentos de categorizar no hacen más que distorsionar los seres y fenómenos que se ubican en las intersecciones. Lugones señala la necesidad de"reconceptualizar la lógica de la intersección" superando con esto el pensamiento categorial, ya que "solo al percibir género y raza como entramados o fusionados indisolublemente, podemos realmente ver a las mujeres de color" (79: 2008).

\section{Marcos de política}

Los marcos de política son entendidos por Mieke Verloo como un"principio organizador que transforma la información fragmentaria o incidental en un problema de estructura y sentido, en la que una solución está implícita o explícitamente incluido". Verloo es tajante al señalar que los marcos de política no son descripciones de la realidad, sino más bien son construcciones que dan sentido a esta, permitiendo su comprensión (2005: 20).

La autora señala que un marco de política tiene un formato típico, conectado a la política y a su formulación. Asimismo, señala que existe una representación implícita o explícita de un diagnóstico, conectado a un pronóstico y a un llamado a la acción (Verloo, 2005: 21).

Siguiendo la misma línea, existen al menos dos dimensiones fundamentales de un marco político: el "diagnóstico" y el "pronóstico" de un problema (Verloo, 2005), pero también hallamos otras dimensiones no menos importantes de considerar en el análisis, entre las que encontramos: "Ia voz, los roles en el diagnóstico y el pronóstico, el género y la interseccionalidad, ubicación, mecanismos, y el equilibrio entre las diferentes partes de un texto de política" (Verloo y Lombardo, 2007).

Las diferentes interpretaciones inciden en la construcción de los problemas políticos (Bacchi, 1999). Esta situación afecta no solo al problema sino también a las soluciones que se dan para abordarle. En este sentido, el análisis de marcos de política puede contribuir a la identificación de la coherencia entre un problema y su solución, y cómo ambos elementos están presentes en los textos políticos (Verloo y Lombardo, 2007).

Uno de los aspectos relevantes de este enfoque es la identificación de las ausencias en el discurso político, además, permite identificar prejuicios "que pueden, sin proponérselo formar los discursos políticos y, en consecuencia, se puede revelar inconsistencias latentes, o incluso los prejuicios de género, integrado en el diseño de políticas públicas" (Verloo y Lombardo, 2007). Asimismo, este tipo de análisis permite identificar las exclusiones existentes en la formulación de las políticas, visibilizando la forma en que "las estrategias discursivas pueden modificar el proceso en sí mismo por medio de la exclusión de algunos actores del debate" (Triandafyllidou y Fotiou, 1998: 6 y 4; citado en Verloo y Lombardo, 2007).

\section{Supuestos orientadores del trabajo}

La investigación desarrollada está asentada en dos supuestos centrales relacionados con el análisis de marcos interpretativos de política y con la interseccionalidad 
de género, etnia y raza. El planteamiento hipotético ha recibido la influencia de los supuestos contemplados en la investigación desarrollada por el proyecto MAGEEQ, al que se ha hecho referencia anteriormente. Así, para los efectos de esta investigación se plantea como primer supuesto que la existencia de diferentes marcos interpretativos sobre las mujeres migrantes afecta a la formulación de las políticas migratorias implementadas por la Comunidad de Madrid. En una segunda hipótesis se plantea que los marcos de política presentes en los planes de integración analizados tienen un escaso carácter interseccional, dejando de lado las consideraciones a las desigualdades de género, raza y etnia.

De esta forma, los planes de integración diseñados por la Comunidad Autónoma de Madrid (2006-2008 y 2009-2012) contendrían variadas interpretaciones y definiciones que atañen a las mujeres migrantes, construyéndose a partir de ellos una política migratoria fundamentada en marcos que posicionan a las mujeres migrantes como víctimas, vulnerables y subalternas. Lo anterior, tiene relación con la presencia de un discurso que ha victimizado a los diferentes colectivos de migrantes (Pedone, 2004). Discurso en el que se crean y reproducen ciertos estereotipos y representaciones de las mujeres de origen extranjero, relacionándoles con un imaginario que tiende a percibirlas como víctimas de pobreza o como las principales afectadas por redes de tráfico para ejercer la prostitución, entre otras (Fernández, 2006).

\section{Método}

El estudio está centrado en el análisis de marcos políticos de interpretación, de esta forma, los textos políticos constituyen el principal foco investigativo, concentrando en ellos la observación y análisis. Metodológicamente este trabajo considera la estrategia de análisis de marcos interpretativos de políticas. El método policy frame o de marcos interpretativos de las políticas trata de identificar los marcos dominantes y/o en conflicto entre sí en el discurso de los y las actores/as sociopolíticas, marcos que se construyen para dar sentido a diferentes situaciones y acontecimientos, atribuir culpas o causalidad, y sugerir líneas de actuación. El análisis de marcos de política utiliza entre sus estrategias elementos de la grounded theory que incluyen el estudio de "palabras y frases repetidas regularmente a lo largo del texto, de las palabras en su contexto, de las dimensiones de ideas implícitas en los textos, y de cómo las ideas se organizan en diferentes posiciones dentro de estas dimensiones (Strauss y Corbin, 1990; citado en Verloo y Lombardo, 2007).

Verloo (2005) señala que para los fines del proyecto MAGEEQ ha sido relevante considerar la dimensión de la voz de los actores y la atribución de responsabilidad de los mismos tanto en la causa como en la solución del problema. En este estudio dicho análisis cobra un papel preponderante debido a que uno de los objetivos es precisamente estudiar la voz y la atribución de responsabilidad de las mujeres migrantes en las políticas de extranjería que se adoptan, por lo que se considera como estrategia la reformulación de algunos de los puntos centrales revisados por el proyecto MAGEEQ, así como en las preguntas que al respecto se formulara dicho equipo de investigación. 
La utilización de una serie de preguntas guías permite ajustar el análisis de los discursos políticos, contribuyendo a la detección de las inconsistencias presentes en los textos estudiados. De esta manera, "la conciencia de las inconsistencias y las exclusiones en los discursos políticos puede ser una herramienta poderosa tanto para afilar la formulación de políticas de género y los procesos de minimización de la exclusión" (Verloo y Lombardo, 2007). Respecto de las preguntas guías de la investigación aquí desarrollada, se planteó utilizar como pauta general algunas de las interrogantes establecidas en el proyecto MAGEEQ, señaladas por las profesoras María Bustelo y Emanuela Lombardo en el libro Políticas de Igualdad en España y Europa (2007: 99) y publicadas también el sitio web del proyecto.

\section{Tabla 1 PREGUNTAS GUÍAS}

\begin{tabular}{|c|c|}
\hline Dimensión & Preguntas \\
\hline Diagnóstico / Pronóstico & $\begin{array}{l}\text { ¿Qué se representa como problema? } \\
\text { ¿Quérepresentaciones implíitas o explíitas ofrecen los/as actores políticos/as del problema o solución? } \\
\text { ¿Quién o quiénes se supone que tiene/n el problema? } \\
\text { ¿A qué grupo se dirigen las medidas que se toman? } \\
\text { Y si hay un grupo problemático, ¿cuál es el grupo normativo de referencia? }\end{array}$ \\
\hline Dimensiones de género & $\begin{array}{l}\text { ¿Se hace referencia a categorías sociales relacionadas con alguno de los géneros? } \\
\text { ¿Se hacen referencias a comportamientos asociados a las mujeres o a los hombres? } \\
\text { ¿Se mencionan ciertas normas y símbolos sociales asociados a hombres y/o mujeres como parte del } \\
\text { diagnóstico y/o solución del problema? }\end{array}$ \\
\hline Voz & $\begin{array}{l}\text { ¿Quién o quiénes redactan el texto? } \\
\text { ¿Quién tiene voz en los discursos y quién está ausente? } \\
\text { ¿A qué actores/as políticos/as hace referencia el texto? } \\
\text { ¿Qué tipo de fuentes documentales son citadas en el texto? }\end{array}$ \\
\hline Interseccionalidad & $\begin{array}{l}\text { ¿Existe un cruce entre género y otras dimensiones como clase, etnia, raza? } \\
\text { ¿Son considerados cruces entre otras dimensiones? } \\
\text { ¿Se hacen referencias particulares a ciertas etnias o razas? } \\
\text { ¿Qué tipo de representaciones se realizan respecto de las personas extranjeras? }\end{array}$ \\
\hline
\end{tabular}

Fuente: Elaboración propia, basada en Bustelo y Lombardo, 2007: 99.

El enfoque utilizado en este estudio es de carácter constructivista, tomando como premisa la idea de Bacchi (1999) respecto de la construcción de los problemas sociales. Bajo esta perspectiva, el conocimiento no es más que una propuesta que responde a una forma de situarse frente a la experiencia. El estudio presentado está teñido por la subjetividad y la interpretación crítica que realiza la investigadora de los textos analizados, a base de su recorrido profesional y a los elementos expuestos en los anteriores apartados. 
Para un mejor estudio de los textos analizados se utilizaron una serie de preguntas guías tendientes a analizar los discursos políticos, contribuyendo a la detección de las inconsistencias presentes en los textos estudiados. De esta manera, la conciencia de las inconsistencias y las exclusiones en los discursos políticos puede ser una herramienta poderosa tanto para afinar la formulación de políticas de género y los procesos de minimización de la exclusión (Verloo y Lombardo, 2007).

\section{Principales resultados del estudio ${ }^{3}$}

A continuación se exponen aquí algunos de los resultados del estudio realizado, dividiendo la exposición en dos, acorde a cada uno de los textos analizados.

\subsection{Análisis del plan de integración de la Comunidad de Madrid, período 2006-2008}

Frente a la descripción y breve análisis de la decena de ámbitos contemplados en el plan de Integración ${ }^{4}$ 2006-2008 de la Comunidad de Madrid, se ha estudiado el texto político a base de la metodología señalada anteriormente.

El plan tiene como principal objetivo el "promover la integración de las personas inmigrantes que residen en la Comunidad de Madrid" (2006: 5). Fue diseñado por el Instituto Universitario de Estudios sobre migraciones de la Universidad de Comillas, y es el primero de este tipo en implementarse en la Comunidad Autónoma de Madrid (CAM). La metodología utilizada contempló la explotación de fuentes secundarias y los aportes de un grupo de expertos externos, un grupo de apoyo técnico, cuestionarios y entrevistas a profesionales que tenían una relación laboral cotidiana con el colectivo migrante, entre otras acciones. En el texto se menciona que en "múltiples ocasiones se contó con la perspectiva de los propios inmigrantes acerca de su integración" (2006: 9) sin mencionarse la forma en que se realizó esta recopilación de información. Las voz de las personas migrantes no está presente en el documento, menos aún la voz de las mujeres migrantes.

Se observa en sus primeras páginas una primera consideración sobre el tema que aquí se analiza. Señalan que entre las áreas contempladas no se consideró la "cuestión de género", por ser "una realidad transversal considerada de modo explícito en todo y cada uno de los estudios sectoriales" (2006: 7).

En el eje de empleo, se expresa que este se ha convertido en un fin para la población migrante y que es el "primer factor de integración y el primer paso en el proyecto migratorio"

Respecto de los análisis de los textos, en este artículo se señalan solo aquellos elementos que competen a la temática desarrollada.

$4 \quad$ Cabe destacar aquí el uso conceptual de la integración. El enfoque integracionista se encuentra en una posición ambigua entre la idea progresista de la lucha por la igualdad de oportunidades y la teoría de la deficiencia, que acaba explicando los déficits de las minorías desde los propios estereotipos de estas. Para muchos teóricos y teóricas sigue constituyendo una forma sutil de racismo y una creencia en la superioridad de la cultura receptora (Muñoz, 2001). 
(2006: 39). El diagnóstico del área empleo evidencia la segmentación sectorial del mercado de trabajo y las diferencias ocupacionales por razón de nacionalidad (2006: 48). En relación con la situación de las mujeres se señala la creciente demanda de mano de obra no cualificada de mujeres extranjeras, la que se produce en un"contexto determinado por la creciente incorporación de la mujer al mercado de trabajo, el progresivo envejecimiento de la población y el todavía insuficiente carácter de las prestaciones públicas" (2006: 48). Así, el trabajo de las mujeres migrantes se concentra"o bien en tareas domésticas en su propio hogar o bien en servicios domésticos, aspecto que, unido a la concepción que se tiene de este tipo de trabajos, acentúa la marginación de este colectivo" (2006: 48).

Ante tales datos, el equipo redactor del plan establece que las mujeres migrantes se encuentran en una situación de "especial vulnerabilidad", debido a que "ven delimitada su actividad profesional mayoritariamente a sectores específicos como el servicio doméstico, junto con la existencia de una infravaloración de este tipo de trabajos que acentúan la situación de marginalidad en la que se encuentran" (2006: 51). Frente a este escenario, se propone como objetivo el "Promover el empleo de las mujeres migrantes", cuya única acción es la "implantación de un programa específico de empleo para favorecer la inserción sociolaboral de las mujeres inmigrantes víctimas de violencia de género" (2006: 176).

Esta primera observación evidencia la inconsistencia entre el diagnóstico de la situación de empleabilidad de las mujeres migrantes y la prognosis realizada. El marco político indica que las mujeres requieren de una acción que fomente su empleabilidad siempre y cuando sean víctimas de violencia de género. La promoción del empleo como solución dista del diagnóstico expuesto.

En las actuaciones no se hace mención a ninguna instancia que permita modificar las situaciones de discriminación por género y etnia que viven las personas migrantes. Tampoco se señalan instancias que les permitan solucionar las diferencias entre los niveles formativos y la baja cualificación requerida para los trabajos desempeñados.

En relación con las dimensiones de género en el texto, se visualiza a las mujeres migrantes como sujetos frágiles, proclives a la exclusión y marginación social, debido a sus mayores dificultades para adaptarse al nuevo contexto. Son observadas como "otras culturales" propensas a la vulneración en sus derechos debido a que esto sería lo esperable en los contextos culturales y tradiciones en origen (mutilación genital, violencia de género, asimetría en las relaciones de pareja, desconocimiento de derechos sexuales y reproductivos), lo que tácitamente está relacionado con la idea de "subdesarrollo"y "retraso cultural". Esto puede visibilizarse en la aseveración realizada respecto de que los problemas en los embarazos de mujeres migrantes suelen darse por "el escaso hábito de realizar controles en el embarazo en sus países de origen", a lo que se sumaría el desconocimiento de los programas perinatales de la CAM y de la accesibilidad a ellos (CAM, 2006: 108).

Cabe mencionar también que el texto está redactado íntegramente en un lenguaje masculinizado. Y que a pesar de que en un inicio se compromete a tomar en cuenta 
el género como una variable transversal, no está presente en todas las categorías, sin considerarle ni en el diagnóstico ni en la solución del problema.

Respecto de la dimensión de interseccionalidad se destaca que existen en algunas ocasiones cruces entre género y etnia, pero solo en los niveles de diagnóstico, lo que no es extensible a las soluciones propuestas. No se hacen cruces con otras dimensiones como diversidad sexual o discapacidades.

En relación con las mujeres migrantes víctimas de violencia de género, el informe señala que casi un tercio de "las denuncias de malos tratos producidos por la pareja o expareja han sido puestas por mujeres migrantes" $(2006,109)$ y que esto hace absolutamente necesario entregarles una cobertura sanitaria acorde a sus necesidades. El plan contempla para ellas la "información y orientación (...) mediante recursos de acogimiento temporal, orientación jurídica y atención psicosocial"y "acciones dirigidas a la atención de mujeres inmigrantes con problemas de desestructuración familiar o personal" $(2006,187)$. Sin embargo, no se hace mención a la situación particular de cada mujer en relación con la residencia, la que en el contexto español del año 2006 era de vital importancia para realizar cualquier acción, ya que las mujeres no contaban con el reconocimiento de víctimas de violencia si su situación de residencia no estaba regularizada.

Respecto del tráfico de personas, no existen antecedentes diagnósticos en el texto. No hay referencias a datos, cifras, estadísticas o caracterización. A pesar de esto, se establecen acciones de acogimiento temporal "puesto a disposición de las necesidades de las mujeres inmigrantes que se encuentren en condiciones de abandonar las redes de tráfico de personas con fines de explotación sexual" $(2006,187)$. Esta medida no toma en consideración la casi imposibilidad de que las mujeres inmersas en las redes de tráfico puedan salir por sí solas de las mismas. Por tanto, se manifiesta una dificultad entre el diagnóstico realizado y la solución otorgada a la problemática, la que no tiene sustento al no considerar los aspectos involucrados en las situaciones de tráfico de personas. No es la única actuación respecto del tema. La CAM compromete acciones en el ámbito del codesarrollo, destinando recursos en los países de origen de mujeres víctimas de tráfico de personas. Sin embargo, la dificultad es la misma, no existe diagnóstico al respecto por lo que es difícil conocer el lugar al que se derivan tales recursos y la posible efectividad de tal actuación.

En este sentido, existe una construcción de las mujeres migrantes como posibles "víctimas" de tráfico de personas, lo que se ajusta a la relación prejuiciosa que se realiza entre los movimientos migratorios de mujeres y el comercio/violencia sexual. Situación similar sucede en el eje salud, en el que se asevera que las mujeres migrantes "que trabajan en el ámbito de la prostitución" exponen al colectivo migrante "a riesgos de infecciones por enfermedades de transmisión sexual" (2006: 107), lo que no es apoyado por dato alguno o cita que evidencie lo afirmado.

También es señalado que "las pautas de comportamiento sexual (...) tienden a venir condicionadas por la posición de la mujer en relación al varón en función de los distintos 
patrones culturales" (2006: 107) y que las mujeres migrantes "muestran cierto desconocimiento en relación con temáticas de salud sexual y reproductiva". Estos juicios son realizados a pesar de que se reconoce que faltan datos en relación con lo señalado, aun así, la CAM realizaría esfuerzos para "erradicar los estereotipos negativos de género y la igualdad de derechos sobre el cuerpo y la sexualidad entre mujeres y hombres". De tal manera, se observa la existencia de un prejuicio respecto de los estereotipos sexuales presentes en la población migrante. Cuando el texto hace referencia a la posición de la mujer en relación con el hombre la está situando implícitamente en una posición de desventaja y sumisión. En las actuaciones es posible encontrar un plan de educación sexual y salud reproductiva orientada a mujeres jóvenes, no así en adultas.

En el ámbito de la "participación y gestión de la diversidad" el diagnóstico no apunta a situaciones particulares asociadas a la participación de mujeres, sin embargo, en las actuaciones se establecen objetivos parta "impulsar el asociacionismo de las mujeres migrantes a través de programas de asociaciones de inmigrantes, organizaciones de apoyo para el fomento del asociacionismo entre mujeres y la celebración de encuentros anuales entre las asociaciones de mujeres inmigrantes y organizaciones de apoyo para el intercambio mutuo de conocimientos y experiencias, realizando exposiciones y actividades"(2006: 198).

Respecto del diagnóstico presentado por la CAM en el ámbito de los servicios sociales se presenta a la población migrante como carente de recursos y vulnerable (2009: 83). Las actuaciones diseñadas para este ámbito dan cuenta de que hay antecedentes que no son expuestos en el diagnóstico, pero sí son considerados en las acciones contempladas (2006: 186, 178, 229).

Respecto de la participación, se plantean objetivos destinados al fomento de la participación de las mujeres en asociaciones, sin contar con que según los estudios son las mujeres quienes tienen mayor participación en las asociaciones de migrantes. Además no se consideran otros espacios de participación que sean externos a la participación en estas asociaciones, lo que sí podría generar una mayor integración, como la participación en agrupaciones vecinales, o en las AMPAS de los colegios u otras agrupaciones en las que sí se pueda vivenciar la interculturalidad. Mediante este actuar pareciera fomentarse la reunión entre migrantes nada más.

Respecto al eje "Familia", el diagnóstico es rico en cuanto a las intersecciones entre género y etnia-nacionalidad (2006: 86). Sin embargo, no se realizan actuaciones al respecto. No hay acciones tendientes a la modificación de pautas culturales y sociales de asimetría en las relaciones entre hombres y mujeres al interior de las familias, estas son asumidas dentro de la diversidad sin proponer cambios a este tipo de situaciones. Tampoco existen referencias en torno a la solución o mitigación de los problemas asociados a la existencia de familias monoparentales en el cuidado de hijos e hijas reagrupados.

Respecto de los marcos de política, en el texto se aprecia un marco relacionado con la visión de la mujer migrante vulnerable. En el documento se relaciona la vulnerabilidad 
de las mujeres migrantes con su posición en el mercado de trabajo y con la presencia de estas en las estadísticas de violencia de género.

En el ámbito de los recursos sociales, se observa la prevalencia de este marco. Esta representación se nutre de una serie de estereotipos y prejuicios presentes en el texto situando a las mujeres migrantes como víctimas de malos tratos, tráfico de personas con fines de explotación sexual o susceptibles de mutilación genital, sin especificar las determinantes ni las condicionantes de cada situación específica.

El marco presentado es reforzado cuando se habla de codesarrollo; deja de lado el capital social y cultural existente en las relaciones trasnacionales que establece la población migrante con su familia y vínculos en origen, sin presentar la importancia que tienen las mujeres en las economías locales, ni su poder ni fuerza en la toma de decisiones familiares y locales. Solo se hace referencia a ellas en cuanto a lo beneficioso que es para la CAM el codesarrollo en área social en tanto se generarían "Mayores oportunidades para las mujeres extranjeras y también nativas (empleo de inmigrantes en sector servicios doméstico y de proximidad), lo cual permite aumentar la conciliación laboral y familiar" (CAM, 2006: 146). Conciliación que no se da en el caso de las mujeres migrantes.

Existe también otro marco de política denominado "lo familiar es privado". En él las relaciones asimétricas entre hombres y mujeres en la familia son asumidas como un asunto cultural y social propio y privado, justificando y revitalizando la división sexual del trabajo. En este sentido, en el texto se observa una imagen estereotipada de las mujeres en relación con la responsable del cuidado familiar, lo que es reflejado al hablar de la alimentación de las familias, responsabilizándoles del tema: "la mujer suele ser la persona de referencia encargada de la alimentación familiar y, algunos estudios, apuntan el posible factor de riesgo existente entre la población inmigrante en relación con la alimentación cuando no se da la presencia de la figura de la mujer" (CAM, 2006: 108). De esta forma, se refuerza la función reproductiva asociada tradicionalmente a las mujeres, acorde a la división del trabajo, por encima de factores productivos o de inserción en la sociedad de acogida.

Así también, recalcan la necesidad de considerar en los análisis "la importancia de las relaciones de género (...) algunas mujeres proceden de países donde las relaciones asimétricas de género son más acusadas, hecho que repercute de manera considerable en la vida de estas mujeres" (2006, 159), esto hace que "los roles y responsabilidades del hombre y la mujer dentro de las dinámicas de las familias inmigrantes varían según predominen los modelos familiares patriarcales o matriarcales", afirmación que realizan sin mayor evidencia. Este tipo de aspectos junto a la diversidad de estructuras familiares "deben ser tenidos en cuenta para favorecer el desarrollo de estos niños y niñas, evitándose así las consecuencias de la desigualdad y la discriminación" (2006: 160). Sin embargo no existen soluciones relacionadas con los puntos mencionados en el diagnóstico.

Resulta interesante lo que sucede en términos de la consistencia entre el diagnóstico y pronóstico, y el prejuicio de género asociado a las situaciones de mutilación genital. En el documento no se hace referencia diagnóstica al respecto. En el apartado 
correspondiente a salud, la situación no es tratada, tampoco se hace en lo correspondiente a servicios sociales. Sin embargo, en las partidas presupuestarias se dedican tres apartados al tema en específico. Por un lado, se destinan dineros a una campaña dirigida a la prevención de la mutilación genital femenina, orientada a profesionales tratantes, así también se destinan recursos a cursos de formación para profesionales sanitarios y, por otro, se contempla el "diseño y puesta en marcha de un programa específico dirigido a prevenir la mutilación genital femenina como un acto de violencia contra las mujeres constitutivo de delito, difundiendo sus graves consecuencias físicas y psíquicas sobre la salud" (CAM, 2006: 188, 229).

Para finalizar esta sucinta presentación del análisis realizado se debe mencionar la inexistencia en el texto de acciones de promoción de la autonomía de las mujeres o apoyos en la conciliación. De esta manera, los problemas son detectados pero no se impulsan instancias de solución.

\subsection{Análisis del plan de integración de la Comunidad de Madrid, período 2009-2012}

En el caso del plan de integración 2009-2012, y a diferencia del anterior, el género no es un asunto transversal. Las situaciones y condiciones relativas a las mujeres (y por ende a los hombres) son englobadas en el eje "Familia" de este plan. Así, cada una de las temáticas que les atañen está englobada en el contexto familiar, considerada por el equipo que redacta el plan como la unidad básica de la sociedad.

Todo lo que tiene relación con la sexualidad es atribuida a las mujeres, no hay referencias a los hombres en las medidas de prevención de enfermedades de transmisión sexual, o el comercio sexual, etc. Las mujeres en este plan están sexualizadas, los hombres no. Se menciona también que las mujeres migrantes están insertas en culturas que fomentan y validan los malos tratos, en una mirada extremadamente generalizante del fenómeno en cuestión.

Las mujeres migrantes son representadas como víctimas: de los malos tratos y de violencia sexual. Se les considera además como vulnerables solo por el hecho de ser mujeres y migrantes, lo que amerita un tratamiento diferenciado a sus particulares necesidades.

Se les considera responsables de las situaciones que las aquejan, ejemplo de esto es la aseveración de que la presencia de dificultades en el embarazo tiene relación con hábitos de atención sanitaria tardía, lo que habría sido adquirido en la experiencia anterior con el modelo de salud de sus respectivos países de origen, remarcando "un escaso hábito de realizar estos controles en los países de procedencia, pero también, la menor adherencia a los criterios de seguimiento del embarazo refleja problemas de información y adaptación cultural" (2009: 170).

En relación con la violencia contra la mujer, se plantea que la violencia de género hacia mujeres migrantes se debe abordar desde el género y desde la cultura. De esta 
manera "se hace necesario realizar un tratamiento especial por las características particulares de cada nacionalidad por razones de edad, país de procedencia, cuestiones culturales, entre otras consideraciones" (2009: 229).

Se establece también que las mujeres migrantes sufren una "doble vulnerabilidad por ser mujery por ser inmigrante, haciéndose hincapié en los últimos tiempos en la necesidad de integrar la perspectiva de género en todas las políticas migratorias. En el caso de las mujeres inmigrantes irregulares, estarían ante una situación de triple vulnerabilidad" (2009: 229).

En este sentido, el plan vuelve a señalar la "vulnerabilidad de las mujeres migrantes" solo por el hecho de ser tales, debido a una serie de situaciones y circunstancias que "aumentan su vulnerabilidad en relación con los malos tratos, existe un reconocimiento a favor de que las mujeres inmigrantes afectadas de una doble vulnerabilidad tengan un tratamiento diferenciado y la perspectiva de género se integre en todas las políticas migratorias" (2009: 231).

Ante esto, el equipo redactor del plan señala que en "aquellos grupos en los que la cultura es claramente patriarcal, se corre el peligro de volver a la reclusión de las mujeres en el espacio privado. De esta manera se traslada al país de acogida los modelos de discriminación existentes en el lugar de origen" (2009: 231). Al respecto, señalan que en el caso de la migración de mujeres procedentes de áfrica, su llegada al país se produce luego de la reagrupación de sus parejas, siendo legal y económicamente dependientes de quien les reagrupa. El equipo señala que en caso de violencia de género las mujeres tendrían un "gran temor a denunciarlo por miedo a perder la red social y padecer el desarraigo porque la violencia sufrida, normalmente, no desaparece en el país de acogida". (2009: 231).

Esta situación se daría menos en el caso de las mujeres de origen latinoamericana, ya que "están presentes en el ámbito externo, dependen menos de sus parejas y vienen de sociedades donde los divorcios tienen cauces legales. Además, por lo general, serían ellas las que inician el proceso migratorio, obteniendo la regularización. Ello les haría más proclives a denunciar situaciones de malos tratos"(2009: 231). Sin embargo, estos juicios de valor respecto de las mujeres latinoamericanas no son fundados en estudios ni estadísticas al respecto.

Respecto de las culturas de origen y su relación con la violencia, el equipo redactor del plan tiene una particular visión en la que se señala que, en origen, las culturas "presentan diferentes valoraciones en cuanto a la relación entre los sexos, la que no se desarrolla de forma igualitaria, en la práctica y desde el modo conceptual del papel que socialmente se concede a uno y a otra". A continuación se menciona la habitualidad de los"malos tratos físicos y psíquicos a los que son sometidas algunas mujeres, las que les ocasionan graves perjuicios en su autoestima y dignidad personal". En este sentido, el plan atribuye al proceso migratorio de las mujeres el inicio de "procesos de independencia económica, incrementándose su poder de decisión y negociación frente a sus parejas" (2009: 231). 
De esta forma, el plan, sin presentar antecedentes que fundamenten la idea, deja entrever la existencia de dos tipologías de mujeres migrantes: por un lado, la mujer latinoamericana, independiente, con mayores posibilidades de estar regularizada; y por otro lado, la mujer de procedencia africana, más sometida y dependiente. Esta situación puede darse debido a que la legislación española facilita la regularización y nacionalización de personas extranjeras nacidas en alguna excolonia española, lo que no se produce con las personas de origen africano, quienes son mayormente discriminadas por sus dificultades administrativas en la regularización.

El plan de integración hace referencia a la explotación sexual de mujeres inmigrantes. Señalan que esto tiene relación con una cantidad de variables que han de ser consideradas en su tratamiento:

\begin{abstract}
"Al abordarse este tema, se debe tener en cuenta que la pobreza, la dependencia, una educación inadecuada, la adicción a drogas, la falta de vivienda, la discriminación racial y sexual son temas que aparecen en las personas que están siendo o han sido prostituidas y por tanto, en la mayoría de las ocasiones, los malos tratos, las carencias afectivas, el analfabetismo, las drogodependencias, las condiciones vigentes del fenómeno migratorio y las graves necesidades económicas son circunstancias que favorecen el ingreso en el mundo de la explotación sexual" (2009: 232).
\end{abstract}

Sin embargo, la declaración realizada aquí deja fuera a los factores de dominación que inciden en la explotación sexual de mujeres. Pareciera que se quiere dejar en manos del contexto y de factores de riesgo la explotación de mujeres, cuando en muchos casos tiene relación con el tráfico de personas, la violencia sexual, más que con situaciones contextuales.

En el plan se define el tráfico de personas como una violación a los derechos humanos, afirmando que la trata "supone una transacción con la persona entendida como un objeto, que exige, además de un movimiento, unos fines de explotación de las personas víctimas de la trata que puede ser laboral, sexual u otro tipo". Siendo esta una de las manifestaciones "más brutales de la violencia de género" (2009: 232).

Las soluciones y prognosis propuestas en el plan son coherentes con las citas anteriormente revisadas, existiendo todo un apartado que hace referencia a la lucha contra la explotación sexual y apoyar a la mujer migrante en riesgo social (2009: 274). Se destaca la existencia de medidas de conciliación laboral y familiar orientadas a la población migrante, medida que no estaba presente en el plan anterior (2009: 272). Sin embargo, hemos de señalar también que la prognosis responde a tres visiones de mujeres: víctimas de violencia de género, víctimas de explotación sexual, y mujeres que presenten necesidades de conciliación laboral-familiar (en una medida marginal en comparación con las otras dos representaciones). La mujer en el plan responde a esos tres estereotipos y siempre en el contexto de familia.

En el transcurso del texto se identifican algunos marcos de política relacionados con las mujeres migrantes. Un primer marco es la mujer migrante como vulnerable 
por el hecho de ser tal, marco de interpretación en la que existe una representación de las mujeres migrantes como susceptibles de abusos y, por tanto, necesitada de protección y asistencia por parte de la administración. En este marco se hace evidente la consideración de la población migrante en general como una minoría étnica, la que complementada con el género constituye un mecanismo cultural que les niega protagonismo y las etniciza en términos culturales.

Además, se denota la existencia de un marco de política que posiciona a las mujeres migrantes como subalternas, sumidas en un modelo tradicional de mujer con pareja transnacional o no, dependientes, pasivas y limitadas al espacio doméstico. Esta mujer migrante está contextualizada en el marco de la reagrupación familiar, la que además la hace dependiente de una pareja que determina el proyecto migratorio y las opciones de integración que esta podría tener. Este marco ignora también los itinerarios diversos de las mujeres, y la posibilidad de generar sus propios proyectos migratorios. Ignora también la gran cantidad de mujeres que han sido pioneras en la migración. Además, se dejan de lado las iniciativas de las mujeres como trabajadoras y emprendedoras, invisibilizando y ocultando la importancia de las mujeres en el desarrollo económico de sus naciones de origen y en el orden mundial.

Se aprecia la presencia de un marco de homogeneización, en el que las mujeres son el símbolo de la otredad cultural. Pero esto también cabe para el resto de la población migrante, la heterogeneidad de las mujeres migrantes es evidente, pero esto no se recoge en el plan analizado.

Los planes de integración realizados en la Comunidad de Madrid poseen una visión respecto de las mujeres migrantes, la que las representa como sujetos frágiles, proclives a la exclusión y marginación social. Las mujeres de origen extranjero, por el solo hecho de serlo son observadas como "otras culturales", propensas a la vulneración de sus derechos.

\begin{abstract}
"... parece cierto afirmar que a las mujeres inmigrantes, por el hecho de ser tales, les acompañan una serie de circunstancias que aumentan su vulnerabilidad en relación con los malos tratos (...) Por ello, existe un reconocimiento a favor de que a las mujeres inmigrantes afectadas de una doble vulnerabilidad tengan un tratamiento diferenciado" (CAM 2009, 231).
\end{abstract}

Estas afirmaciones centran su mirada en una sola imagen de migrante, sin considerar la multiplicidad de orígenes y contextos de las mujeres. Se construye a su vez una imagen de la mujer migrante, estereotipada, heterosexual, y afectada por el proceso migratorio: "muchas de las mujeres inmigrantes se encuentran aisladas sin el apoyo o la información suficientes para intentar salir de la situación de maltrato en que viven"; señala a su vez que las mujeres suelen estar más cercanas a "situaciones límite de mayor precariedad, soledad, desarraigo y una cultura patriarcal más consolidada hacen que la violencia doméstica afecte a las mujeres inmigrantes en mayor medida que a las mujeres autóctonas"(2009: 232).

En el ámbito de "Mujer, Familia y Juventud", se afirma que "la familia es la primera institución a la que pertenecemos" (2009: 222) y que el contexto de la globalización ha 
modificado las pautas y dinámicas familiares tradicionales. De esta forma, y en relación con las familias de migrantes, "es obligado recordar que las realidades familiares de las personas inmigrantes son muy diversas y no responden a un patrón único" (2009: 223). A base de esto se plantea la importancia de considerar la transnacionalidad en el análisis de estas familias, lo que resulta absolutamente necesario para la comprensión de las dinámicas y las relaciones de género que en estas se producen.

Se menciona además que la existencia de personas migrantes en situación de irregularidad administrativa les expone a la "indefensión, vulnerabilidad e incertidumbre" (2009: 225). Condicionamientos que les conducirían a vivir en condiciones de "precariedad, desestructuración familiar y en situaciones de exclusión familiar".

El plan 2009-2012 supone un intento de superar las deficiencias del primer plan de integración implementado. A pesar de lo anterior se observan ciertas situaciones y juicios que presentan representaciones sobre la migración y particularmente sobre las mujeres migrantes que deben ser tomadas en consideración.

En primer lugar, el texto está redactado en una voz masculina, excluyente, lo que echa por tierra los intentos señalados de incluir la perspectiva de género en las políticas implementadas. No se consideran cruces con otras dimensiones como discapacidad, opción sexual, entre otras.

La gran mayoría de los datos son desagregados por "sexos", concepto que es utilizado para hacer referencia cuando se desagregan los datos diferenciando a hombres de mujeres. En muchos de los datos presentados no se señala el total de migración que ha recibido tal o cual servicio o beneficio, sino que hay un desglose inmediato de las nacionalidades con mayor cantidad de personas atendidas.

Existe un dato que no es reforzado estadísticamente y que es interesante de destacar, que es la no escolarización de algunas niñas señalando que esto se agrava según la nacionalidad de origen (2009: 149). Este en un tema a trabajar en futuras investigaciones sobre el tema.

Existen juicios sobre la precariedad de la situación de las personas migrantes, en una mirada que claramente es homogeneizante. Estas situaciones de precariedad les harían vulnerables a problemas de salud. Asimismo, se señala que los y las migrantes tendrían "condiciones de trabajo más penosas de lo habitual" (2009: 168), debido a los trabajos a los cuales acceden. Estos juicios están presentes a lo largo del plan mencionado y fomentan una representación de la población migrante como carenciada y necesitada.

Se presta bastante atención a la Mutilación Genital Femenina, aunque se reconoce su baja incidencia y la necesidad de trabajo preventivo en origen a partir de acciones de cooperación (2009: 170). Esta atención viene a reforzar las diferencias culturales con colectivos a los que se quiere presentar en esa "otredad" señalada con anterioridad, y reforzar a cierto tipo de mujeres migrantes como potenciales víctimas de abusos a 
pesar de que podrían no serlo. Se refuerza también la idea de que las migrantes provienen de países y culturas de menor "desarrollo", por cuanto es posible que repliquen este tipo de prácticas en las sociedades de acogida. No se explicita el perfil de mujeres potenciales víctimas de MGF, ni sus orígenes.

Se menciona también que los problemas del embarazo tienen relación con el tardío control del mismo debido al "escaso hábito de realizar estos controles en los países de procedencia" (2009: 170), afirmación del todo generalista y que carece de fundamentación. Además, se responsabiliza de esto a las mujeres migrantes sin tomar en consideración la responsabilidad del sistema sanitario, y del sistema de protección en general, de informar y garantizar los controles necesarios para la población en etapa fértil.

Respecto del ámbito de participación y cultura no se hacen cruces con el género. Esto deja de lado la importancia de las mujeres tanto en la creación de asociaciones de migrantes como en la participación en otras agrupaciones como AMPAS, agrupaciones de vecinos y vecinas, etc. Se deja de lado el fomento de la participación de las mujeres en agrupaciones de mujeres.

Se reconoce el esfuerzo por incluir la noción de transnacionalidad en los análisis realizados. Sin duda esto amplía las dimensiones para comprender los fenómenos asociados a la migración. Además su consideración en cuanto a las relaciones de género es un punto clave en la planificación y ejecución de políticas dirigidas a la población migrante (2009: 223).

Respecto de la violencia de género, se menciona considerar en ella la perspectiva de género, pero también las diferencias culturales. Esto no es lo suficientemente explicitado, como sí lo es en el tema de otros derechos humanos vulnerados. La condena a este tipo de situaciones debiese ser muchísimo más enérgica debido a la magnitud y transversalidad del fenómeno.

Se habla de interseccionalidad aditiva al mencionar la doble y triple vulneración de las mujeres migrantes, sin embargo creemos que dicha consideración no puede darse como una suma de desigualdades, sino como coexistentes la una con la otra, de manera que la una afecta a la otra y viceversa. El plan señala la "vulnerabilidad de las mujeres migrantes" solo por el hecho de ser tales, y que esta adición de desigualdades argumenta la necesidad de contar con un tratamiento diferenciado a sus necesidades y requerimientos.

En el plan se hace referencia a grupos de cultura claramente patriarcal (2009: 231), como si este fuese una característica de algunos grupos migrantes, dejando de lado que la sociedad entera está sumida en el patriarcado. Se señala claramente que las mujeres africanas serían las más sumisas y por tanto vulnerables, sin embargo, esto no se sustenta en más evidencia que en la dependencia legal debido a la reagrupación familiar. Esto no es más que un juicio apriorístico, ya que no hay evidencia que señale 
este dato. Podríamos decir lo mismo de las mujeres católicas o protestante, o de las mujeres de Huelva o Alicante, lo que produciría gran revuelo porque no habría bases suficientes para decirlo, pero aquí el equipo redactor se da la licencia de señalarlo para el caso de las mujeres africanas. Además no se señala qué mujeres africanas, de qué situación socioeconómica o de qué países de procedencia.

Respecto de las remesas, se habla de ellas solo en el ámbito económico, sin hacer análisis de género, ignorando la importancia de las mujeres migrantes en las economías nacionales e internacionales.

El plan carece de consideraciones hacia la agencia de las mujeres migrantes, así como del capital social y cultural que estas tienen. La mirada sigue siendo victimizante. Los temas tratados en el ámbito que les compete son violencia de género y violencia sexual. No hay perspectiva que potencie su autonomía y los emprendimientos como estrategias de integración a la sociedad de acogida.

\section{CONCLUSIONES}

En los análisis realizados a los dos últimos planes de integración de la Comunidad de Madrid, mediante la metodología de Marcos Interpretativos de Política, se ha identificado la existencia de marcos de interpretación respecto de las mujeres migrantes asentadas en Madrid.

Los planes de integración realizados en la Comunidad de Madrid (2006-2008 y 2009-2012) poseen una visión respecto de las mujeres migrantes que las representa como personas proclives a la exclusión y marginación social. Las mujeres de origen extranjero son observadas como "otras culturales", propensas al atropello de sus derechos. Esto se fundamenta en el prejuicio de que en sus países de origen se habrían "normalizado" las situaciones de abuso y vulneración de derechos, por tanto, y siguiendo este hilo, es esperable que este tipo de situaciones se reproduzcan en las sociedades de acogida.

Las representaciones de las mujeres migrantes existentes en ambos planes las muestran como mujeres subordinadas a sus culturas, a sus relaciones de pareja y pasivas frente al ejercicio de derechos. Estos elementos justifican una institucionalidad que les proteja de potenciales abusos, convirtiéndolas en sujetos altamente vulnerables e indefensas, ubicándolas como beneficiarias de planes y programas acorde a la necesidad de tutela y protección que requerirían.

De esta forma se identifica un primer marco de políticas, el que ha sido denominado para efectos de esta investigación como "mujer migrante vulnerable". En este marco se concibe a la mujer como vulnerable producto de su proceso migratorio. La representación de vulnerabilidad está nutrida por una serie de estereotipos y prejuicios presentes a lo largo de ambos textos. El marco responde a un enfoque de homogeneización y marginación de las mujeres de origen extranjero. 
En este mismo contexto se subinscribe la consideración de las mujeres migrantes como "víctimas". La imagen a la que se recurre es a mujeres susceptibles de abusos y, por tanto, necesitadas de protección y asistencia por parte de la administración.

Este marco responde a una sola concepción de migrante: la de la persona proveniente de países del mal llamado "tercer mundo" o de colonias anteriores, ignorando con esto la migración proveniente de países angloparlantes o la propia migración que ha alimentado la demografía madrileña, es decir, la migración campo-ciudad, y de ciudades a la metrópoli.

Un segundo marco hallado en este estudio, es el de "Mujer subalterna". La subalternidad de la mujer le restringe la voz, y en este marco es representada en el papel de víctima en situaciones de dominación relacionadas con los contextos culturales de origen. Desde esta mirada se afirma que las situaciones de abuso y dominación que podrían darse en origen son reproducidas en las sociedades de acogida. Lo mismo sucede con la repetición de pautas y relaciones de subordinación al interior de la familia. La mujer en este marco no tiene agencia, ni autonomía.

Un tercer marco es el de "homogeneización de las mujeres migrantes", en él las mujeres son el símbolo de la otredad cultural. Se las concibe con características comunes, sin importar su diversidad de orígenes y contextos. Esto es apreciable en los juicios que se hacen respecto de que las culturas de origen son sociedades más retrasadas en el desarrollo, permisivas en los malos tratos y abusos. Se hace evidente la consideración de la población migrante en general como una minoría étnica, la que complementada con el género, constituye un mecanismo cultural que les niega protagonismo y las etniciza en términos culturales. Esto en palabras crea una falsa representación cultural de estos colectivos como un núcleo homogéneo, unitario (Nash, 2008). La heterogeneidad de las mujeres migrantes es evidente, pero esto no se recoge en los planes analizados.

En el estudio ha sido posible observar la complementariedad entre los marcos de políticas aquí expuestos, debido a que todos y cada uno de ellos potencian una concepción política que representa a las mujeres migrantes como personas no susceptibles de la ciudadanía plena. Las representaciones dadas las configuran como dependientes, inferiores, desprovistas de las condiciones necesarias para el pleno ejercicio ciudadano. De esta forma, se promueve una ciudadanía excluyente, que margina a las mujeres migrantes subrayando la subalternidad que se les atribuye.

Las intersecciones no han logrado ser visualizadas como espacios importantes de considerar en el diseño de la política migratoria de la Comunidad de Madrid. Asimismo, las interseccionalidades de género y raza o etnia son insuficientes, ya que los cruces entre las variables no han significado el traer a la agenda la cultura de las "minorías".

La noción de interseccionalidad es presentada solo en el primer plan, haciendo incluso mención a las discriminaciones aditivas, sin embargo, la discriminación es 
relacional. Las múltiples variables han de ser consideradas en políticas como la estudiada, con tal de construir soluciones integrales a las problemáticas diagnosticadas.

Las discriminaciones de todo tipo mandatan a considerarlas como urgentes de tratar en planeamientos autonómicos, pudiendo considerarse las interseccionalidades entre las distintas categorías permitiendo ampliar la mirada. Esto, aunque difícil, tomando en cuenta la posición ideológica del actual gobierno de Madrid, que es la misma del gobierno español, permitiría la construcción de alianzas de significación estratégica. Esta tarea ha sido asumida por los colectivos feministas alternativos, los colectivos de LGTB y las comisiones de algunos barrios emblemáticos de Madrid, quienes denuncian las discriminaciones y que la aditividad solo potencia aún más la visión de marginalidad, creando y recreando un sujeto pasivo hacia el que se dirigen políticas que fomentan la anomia y la dependencia.

La noción de interseccionalidad puede contribuir a un abordaje más real de los problemas en las políticas, planes y programas diseñados, debido a que este enfoque considera que los grupos sociales no son homogéneos, que las personas pueden estar ubicadas en estructuras que capturan relaciones de poder implicadas en estas (patriarcado, racismo, clasismo, heterosexismo, etc.) y que hay efectos únicos no aditivos en estas intersecciones e interacciones.

La no consideración de las desigualdades de género, raza y etnia contribuye a una segregación y exclusión de la ciudadanía plena a las mujeres migrantes en particular, y de la población migrante en general. Las políticas migratorias han construido y fortalecido fronteras, estigmatizando y marcando. Han logrado configurar una forma de ciudadanía incompleta y sesgada, establecida mediante generizaciones y etnoestratificaciones (Pedone, 2004).

Considerar la noción de interseccionalidad constituye un desafío no solo en las investigaciones desarrolladas desde la academia, sino también en la implementación de políticas que superen las nociones de igualdad y permitan las diversidades.

El generar políticas que den cuenta de las diversidades y diferencias, sin dar paso a la exclusión, es una de las grandes tareas, no solo en Madrid, no solo en España, sino en Chile y en todos los rincones.

Las democracias actuales requieren de consideraciones distintas de hacer y vivir la política. Los movimientos sociales de los últimos tiempos nos han señalado que las viejas concepciones e instituciones están en crisis, una crisis que no solo es en lo económico, sino que es una crisis sistémica, una crisis que en palabras de Edgard Morin requiere de humanizar la humanidad (2011: 45). En este sentido, la democracia requiere revitalizarse y considerar la diversidad de sujetos y propuestas. Las sociedades pluriculturales son un hecho constatable.

Las democracias tienen como reto el enfrentar la diversidad cultural, para lo que es absolutamente necesario considerar la dimensión de género, pero no de manera 
sectorial, sino en interrelación con una multiplicidad de dimensiones que han de ser contempladas en los nuevos modelos de democracia que los nuevos contextos requieren.

Desde los feminismos tenemos la responsabilidad de incidir en estas transformaciones. Nunca antes en la historia las mujeres habíamos tenido tal visibilidad y poder político. Pero también nunca antes habíamos tomado tanta conciencia de nuestras diferencias y la importancia de su respeto en las sociedades democráticas, para desde ahí, desde el reconocimiento mutuo, construir, juntarnos en esas tierras medias, donde las identidades son múltiples.

\section{BIBLIOGRAFÍA}

Alonso, José Antonio (2011): "Migración internacional y desarrollo: una revisión a la luz de la crisis". CDP Background Paper No. 11, June 2011. New York: United Nations. [on line] Disponible en: http: //www.un.org/en/development/desa/policy/cdp/ cdp_background_papers/bp2011_11.pdf [Recuperado el 05 de septiembre de 2011].

Anthías, Floya (2006): "Género, etnicidad, clase y migración: Interseccionlidad y pertenencia translocalizacional”. En: Rodríguez, Pilar (Ed.) (2006) Feminismos periféricos. Granada: Alhulia.

Anzaldúa, Gloria (1987): Borderlands/La Frontera: The New Mestiza. San Francisco: Aunt Lute.

Bacchi, Carol (1999): Women, Policy and Politics. The construction of policy problems. Londres: Sage.

Brah, Avtar (2004): "Diferencia y diversificación". En Traficantes de sueños. Otras inapropiables. Feminismos desde las fronteras. Madrid: Traficantes de sueños.

--------- (2011): Cartografías de la diáspora. Identidades en cuestión. Madrid: Traficantes de sueños.

Bustelo, María (2009): Mucho camino por recorrer: la institucionalización de la "interseccionalidad" en España. Ponencia para ser presentada en el IX Congreso de la AECPA: Repensar la democracia: inclusión y diversidad, Málaga, 23, 24 y 25 de septiembre de 2009.

Bustelo, María y Lombardo, Emanuela (2006): "Los 'marcos interpretativos' de las políticas de igualdad en Europa: conciliación, violencia y desigualdad de género en la política". Revista Española de Ciencia Política. Núm. 14, abril 2006, pp. 117-140.

- (2007): Políticas de igualdad en España y en Europa. Afinando la mirada. Madrid: Cátedra. 
Carrizo, Silvia (2006): Mujeres inmigrantes: de la invisibilidad a la presencia. Primer aporte a la reflexión desde la Asociación Malen Etxea [on line]. Disponible en: http: //www. mugak.eu [Recuperado el 18 de mayo de 2011].

CEPAL (2006): Migración Internacional, Derechos Humanos y Desarrollo en América Latina y El Caribe. Montevideo: Trigésimo primer período de sesiones, 20 al 24 de marzo.

Colectivo IOE (1998):"Mujeres migrantes en España. Proyectos migratorios y trayectorias de género". OFRIM, No 3, Suplementos.

Colectivo IOE (2001): Mujer, inmigración y trabajo. Madrid: IMSERSO.

Comunidad de Madrid (2006): Plan de Integración 2006-2008. Madrid: CAM.

(2009): Plan de Integración 2009- 2012. Madrid: Consejería de inmigración y cooperación de la Comunidad de Madrid.

Curiel, Ochy (2011): "La descolonización vista desde el feminismo afro", en Villalba, Cristina y Álvarez, Nacho (2011): Cuerpos políticos y agencia. Reflexiones feministas sobre cuerpo, trabajo y colonialidad. Granada: Ed. Universidad de Granada.

Fernández, Dhayana (2006): "Las mujeres inmigrantes en España. Una realidad compleja”. Las otras miradas, Vol. 6, No 2. Caracas: Universidad de Los Andes.

Hancock, Ange-Marie (2007): "When Multiplication Doesn't Equal Quick Addition: Examining Intersectionality as a Research Paradigm", Perspectives on Politics, 5(1), pp.63-79 [on line]. Disponible en: http://annasampaio.com/uploads/Hancock_2007. pdf [Recuperado el 4 de agosto de 2011].

hooks, bell (2004): "Mujeres negras. Dar forma a la teoría feminista", en Traficantes de sueños. Otras inapropiables. Feminismos desde las fronteras. Madrid: Traficantes de sueños.

Knudsen, Susanne (2006): "Intersectionality - A Theoretical Inspiration in the Analysis of Minority Cultures and Identities in Textbooks". En Caught in the Web or Lost in the Textbook 2006, pp. 61-76, [on line]. Consultado en http: //www.caen.iufm.fr/colloque_iartem/pdf/knudsen.pdf [Recuperado el 23 de junio de 2011].

Lombardo, Emanuela y Verloo, Mieke (2009): "Stretching gender equality to other inequalities: Political intersectionality in European gender equality policies". En Lombardo, Emanuela, Mejer, P. y Verloo, Mleke (eds.) The discursive politics of gender equality. Stretching, bending anda policy-making. Londres: Routledge, pp. 67-84.

(2010): "La interseccionalidad del género con otras desigualdades en la política de la Unión Europea", en Revista Española de Ciencia Política 23/julio, pp. 11-30 [on 
line]. Consultada en http: //www.urv.cat/media/upload/arxius/igualtat/lombardo_verloo.pdf [Recuperado el 01 de julio de 2011].

Lorde, Audre (2003): La hermana, la extranjera. Artículos y conferencias. Madrid: Horas y Horas.

Lugones, María (2004): "Purity, impurity, and separation”. Signs, 19 (2), pp. 458-479.

Lugones, María (2008): "Colonialidad y género", en Tabula Rasa, Núm. 9, julio-diciembre, 2008, pp. 73-101. Bogotá: Universidad Colegio Mayor de Cundinamarca.

Mignolo, Walter (2003): Historias locales/diseños globales. Colonialidad conocimientos subalternos y pensamiento fronterizo. Madrid: Akal.

Mohanty, Chandra Talpade (2002): "Encuentros feministas: situar la política de la experiencia", en Barret, Michèle \& Phillips, Anne (eds), Desestabilizar la Teoría-Debates feministas contemporáneos. México: Paidós.

Moraga, Cherríe y Castillo, Ana (1988): Esta puente, mi espalda. Voces de mujeres tercermundistas en los Estados Unidos. San Francisco: Editorial "Ismo".

Morín, Edgard (2011): La Vía. Para el futuro de la Humanidad. Barcelona: Paidós.

Muñoz, Antonio (2001): Enfoques y Modelos de educación multicultural e intercultural. Madrid: UCM.

Nash, Mary (2004): Mujeres en el mundo. Historia, retos y movimiento. Madrid: Alianza Editorial.

Orozco, Amaia; Paiewonsky, Denise y García Domínguez, Mar (2008): Cruzando Fronteras II. Migración y Desarrollo desde una perspectiva de género. Instituto Internacional de Investigaciones y Capacitación de las Naciones Unidas para la Promoción de la Mujer (UN-INSTRAW), Instituto de la Mujer (Ministerio de Igualdad) [on line]. Santo Domingo, República Dominicana. Disponible en: http://www.remesasydesarrollo.org/ uploads/media/Crossing_BordersII_WEB.pdf [Recuperado el 28 de agosto de 2011].

Oso, Laura (2007): "Migración, género y hogares transnacionales". Actas del 50 Congreso Nacional sobre Migración en España, Valencia, Marzo 2007 [on line]. Disponible en: http: //www.adeit.uv.es/inmigracion2007/index.php [Recuperado el 5 de agosto de 2011].

Parella, Sonia (2003): Mujer, inmigrante y trabajadora: la triple discriminación. Barcelona: Anthropos.

(2005): "Segregación laboral y"vulnerabilidad social" de la mujer inmigrante a partir de la interacción entre clase social, género y etnia", en Solé, Carlota y Flaquer, 
Lluís (eds.) El uso de las políticas sociales por las mujeres inmigrantes. Madrid: Ministerio del Trabajo y Asuntos Sociales, Secretaría de Políticas de Igualdad, Instituto de la Mujer.

Pedone, Claudia (2004): Tú siemprejalas a los tuyos, las cadenas y las redes migratorias de las familias ecuatorianas hacia España. Barcelona: Universidad Autónoma de Barcelona.

Peterson, Elin (2007): “El género en los marcos interpretativos sobre la 'conciliación de la vida familiar y laboral'", en Políticas de igualdad en España y en Europa. Afinando la mirada. Madrid: Cátedra.

Plataforma Interamericana de Derechos Humanos, Democracia y Desarrollo (2008): Migraciones y Derechos Humanos. Primer Informe para la consulta permanente sobre Migraciones y Derechos Humanos en América Latina y el Caribe. Bogotá: Antropos.

Reigada Olaizola, Alicia (2008): "Repensar la crítica feminista desde la frontera: Dilemas y aportaciones en torno al sujeto, la experiencia y la diversidad", en Feminismos en la antropología: nuevas propuestas críticas. XI Congreso de Antropología: retos teóricos y nuevas prácticas [on line]. Disponible en: http://www.ankulegi.org/wp-content/ uploads/2012/03/0606Reigada-Olaizola.pdf [Recuperado el 5 de julio de 2011].

Robert, Elisabeth y López, Diana (2008): Mujeres migrantes en el nuevo orden internacional. ¿Cómo las mujeres se ven especialmente afectadas por las crisis? [on line]. Disponible en: http://www.remesasydesarrollo.org/uploads/media/Ponencia_INSTRAW_28.1108_ TU_MUJER.pdf [Recuperado el 9 de julio de 2011].

Rodríguez, Pilar (Ed.) (2006): Feminismos periféricos. Granada: Alhulia.

Solé, Carlota (2009): Las trayectorias sociales de las mujeres inmigrantes no comunitarias en España. Factores explicativos de la diversificación de la movilidad laboral intrageneracional. Barcelona: Universidad Autónoma de Barcelona, Ministerio de Igualdad.

Spivak, Gayatri Chakravorty (2010): Crítica de la razón poscolonial. Hacia una crítica del presente evanescente. Madrid: Akal.

Suárez Navas, Liliana (2008): "Colonialismo, gobernabilidad y feminismos poscoloniales", en Suárez Navas, Liliana y Hernández, Rosalva Aída (Eds.): Descolonizando el Feminismo. Teorías y prácticas desde los márgenes. Madrid: Ediciones Cátedra.

Trujillo, Gracia (2011):"La rebelión de"las otras" del movimiento feminista: el impacto de la crítica Queer", en Villalba, Cristina y Álvarez, Nacho, Cuerpos políticos y agencia. Reflexiones feministas sobre cuerpo, trabajo y colonialidad. Granada: Ed. Universidad de Granada.

Verloo, Mieke (2005): "Mainstreaming Gender Equality in Europe. A Frame Analysis Approach", The Greek Review of Social Research B' 117, pp. 11-13 [on line]. Disponible en: http: //www.grsr.gr/pdf/117_11-34.pdf [Recuperado el 12 de agosto de 2011]. 
Verloo, Mieke y Lombardo, Emanuela (2007): Contested Gender Equality and Policy Variety in Europe: Introducing a Critical Frame Analysis Approach [on line]. Disponible en: www. ru.nl/publish/pages/.../verloo_multiple_meanings_intro_2007.pdf [Recuperado el 9 de julio de 2011]. 